\title{
GSTM I null genotype may be associated with an increased nasopharyngeal cancer risk in South China: an updated meta-analysis and review
}

This article was published in the following Dove Press journal:

OncoTargets and Therapy

7 September 2015

Number of times this article has been viewed

\author{
Yanni Li ${ }^{1,2, *}$ \\ Wuhanhui Wan ${ }^{1,2, *}$ \\ Ting $\mathrm{Li}^{1,2}$ \\ Jing $\mathrm{Cao}^{1,2}$ \\ Ge $X u^{1,2}$
}

'Department of Otolaryngology, PUAI Hospital, ${ }^{2}$ Tongji Medical College,

Huazhong University of Science and

Technology, Wuhan, Hubei Province,

People's Republic of China

*These authors contributed equally to this work

\begin{abstract}
Although many epidemiologic studies investigated the GSTM1 gene polymorphism and its association with nasopharyngeal carcinoma (NPC) in Chinese, definite conclusions cannot be drawn. To assess the impact of of GSTM1 polymorphism on the risk of NPC, an updated metaanalysis was performed in a Chinese population. A total of nine studies including 1,291 cases and 2,135 controls were involved in this meta-analysis. Meta-analysis of those nine studies showed that GSTM1 null genotype was associated with an increased risk of NPC in South China (odds ratio $[\mathrm{OR}]=1.47,95 \%$ confidence interval [CI]: 1.27-1.70). In subgroup analyses stratified by source of controls, it revealed significant results in population-based studies ( $\mathrm{OR}=1.40,95 \% \mathrm{CI}: 1.19-1.64)$. Additionally, a significant association was found in smokers ( $\mathrm{OR}=3.16,95 \% \mathrm{CI}$ : $1.76-5.67)$. This meta-analysis indicated a marked association of GSTM1 with NPC risk in South China, and there might be an interaction between the polymorphism and smoking on NPC. However, further studies with gene-gene and gene-environment interactions are required for definite conclusions.
\end{abstract}

Keywords: meta-analysis, glutathione S-transferase M1, polymorphism, nasopharyngeal carcinoma

\section{Introduction}

Nasopharyngeal carcinoma (NPC) is considered one of the rarer cancer forms globally, with an estimated 84,400 incident cases and 51,600 deaths in 2008, representing the 24 th most frequently diagnosed cancer form globally and the 22 nd most frequently diagnosed cancer within the developing countries. ${ }^{1}$ However, there is an obvious difference in the distribution of NPC incidence, and the highest incidence rate is in the People's Republic of China and Southeast Asian region. ${ }^{2,3}$ NPC ranked eleventh among all malignancies in the People's Republic of China in 2008, with an incidence rate of 2.8/100,000 person-years in men and 1.9/100,000 person-years in women. ${ }^{3}$ The exact pathogenesis of NPC has not yet been understood up till now. Apart from Epstein-Barr virus infection and exposures to environmental carcinogens, genetic susceptibility seems to be a risk factor playing a crucial role in the development of NPC. ${ }^{4}$

In recent years, several common, low-penetrant genes have been identified as potential NPC susceptibility genes. An important one is glutathione $S$-transferase (GST), which is one of the detoxification enzyme systems and plays an important role in inactivating endogenous and exogenous toxic products under oxidative stress. The GST isoenzymes have been reported to express classes mu, theta, and pi in human lens tissue. ${ }^{5}$ Located on chromosome 1 at 1p13.3, the GSTM1 plays an important role in the xenobiotics' detoxification. The most common genotype of GSTM1 gene is homozygous deletion (null genotype), which has been suggested to be associated with the loss of enzyme activity, increased vulnerability to cytogenetic damage, and results
Correspondence: Ge Xu Department of Otolaryngology, PUAI Hospital, Tongji Medical College, Huazhong University of Science and Technology, 473 Hanzheng Street, Wuhan, Hubei Province 430030, People's Republic of China Email gexu2596@I26.com 
in the increased susceptibility to cancers, including NPC. ${ }^{6,7}$ Meta-analyses of related studies in other ethnic groups have also produced conflicting results due to several factors such as environmental factors, family history, and different genetic backgrounds. ${ }^{8-11}$ Therefore, we conducted an updated meta-analysis to more precisely define the effect of GSTM1 polymorphism on the risk of NPC in the Chinese population only, in an attempt to investigate race-specific effects.

\section{Materials and methods}

The authors advise that the Ethics Committee of Huazhong University of Science and Technology did not require that this study have ethics approval because it is only a metaanalysis rather than an experiment or case-control study.

\section{Search strategy and study selection}

We conducted a systematic literature search for published articles regarding the association of GSTM1 polymorphism and NPC risk. The studies were searched in PubMed, Springer Link, Ovid, Chinese Wanfang Data Knowledge Service Platform, Chinese National Knowledge Infrastructure (CNKI), and Chinese Biology Medicine (CBM) up to January 5, 2015, using the combination of following terms: 1) glutathione- $S$ transferase M1, GSTM, GSTM1; 2) nasopharyngeal cancer, nasopharyngeal carcinoma, or NPC; 3) polymorphism or variant or variation; and 4) Chinese or China or Taiwan. The search was performed without any restrictions on language and focused on studies conducted on humans. In addition to the electronic database search, all reference lists of retrieved articles were manually reviewed to identify additional articles. In our meta-analysis, studies were included if the following criteria were met: 1) if they were case-control or cohort studies describing the association of GSTM1 deletion polymorphism and NPC, 2) if all patients with the diagnosis of NPC were confirmed as having the disease by pathological or histological examinations, 3) if there was a clear description of GSTM1 polymorphism in NPC patients and controls, and 4) if all participants were Chinese. The reasons for exclusion of studies were: 1) duplicate publications, 2) incomplete data, 3) no control, 4) meta-analyses, letters, reviews, or editorial articles.

\section{Data extraction}

Two investigators independently extracted information from all eligible publications according to the inclusion criteria listed in the earlier section. Disagreements were settled out by discussion among all reviewers. The title and abstract of all potentially relevant articles were screened to determine their relevance. Full articles were also scrutinized if the title and abstract were ambiguous. We extracted standardized data sets from studies of GSTM1 polymorphism and NPC. The following information was sought from each publication: the first author, publication year, source of controls, geographic area, sample size, and the number of subjects with two GSTM1 genotypes. In this meta-analysis, the quality assessment of each individual study was conducted according to the nine-star Newcastle-Ottawa Scale. ${ }^{12}$

\section{Statistical analysis}

The strength of the association between GSTM1 null allele and risk of NPC in Chinese was measured by crude odds ratios (ORs) with $95 \%$ confidence intervals ( $95 \% \mathrm{CIs}$ ), and the significance of the pooled OR was determined by the $Z$ test. Given that there was distribution of null/present heterozygote in only one study selected, ${ }^{13}$ the Hardy-Weinberg equilibrium (HWE) test could not be conducted. Cochran's $Q$-statistic was used to assess between-study heterogeneity, and a significant $Q$-statistic $(P<0.10)$ indicated heterogeneity across studies. If there was heterogeneity, then the random-effects model was chosen to pool the OR with $95 \% \mathrm{CI}$, otherwise the fixedeffects model was used. Sensitivity analysis was conducted to verify stability of the meta-analysis using both models (the fixed-effects model and random-effects model). Publication bias was investigated with the funnel plot, in which the standard error (SE) of log OR of each study was plotted against its OR. Funnel-plot asymmetry was further assessed by the method of Begg's and Egger's tests. Moreover, subgroup analyses were performed to test whether the effect size varied by the smoking status and the source of control population. All statistical analyses were conducted using Stata version 10.0 (Stata Corp, College Station, TX, USA). A two-sided $P<0.05$ was considered statistically significant.

\section{Results Description of included studies}

A total of 22 articles that examined the association between GSTM1 polymorphisms and risk of NPC were identified. After screening the titles and abstracts of these articles, nine were excluded. Of the remaining 13 potentially relevant articles, three were excluded because they concerned subjects included in an expanded series and one in North China ${ }^{14}$ was also excluded due to the possible bias from geographic areas. The flowchart of study selection is shown in Figure 1. Finally, nine case-control studies ${ }^{13,15-22}$ including 1,291 NPC cases and 2,135 controls were involved in this meta-analysis, which evaluated the relationship between GSTM1 polymorphism and NPC risk. The source of controls was mainly based on 




Figure I Flowchart of the literature search.

a healthy population. The geographic areas were all in South China. The characteristics of the included studies are summarized in Table 1.

\section{Quantitative data synthesis} Overall analysis

There was no evidence of between-study heterogeneity in all included studies $\left(\chi^{2}=11.58, P=0.171\right)$. Therefore, the fixed-effects model was used in overall analysis. The results showed that the pooled OR with 95\% CI for NPC in South China with null GSTM1 was $1.47(1.27-1.70, P=0.000)$ (Figure 2A). In addition, the finding from cumulative metaanalysis showed that there was a trend of more obvious association between the GSTM1 null genotype and risk of NPC in South China based on the data accumulated by publication year (Figure 2B).

\section{Subgroup analysis}

In the subgroup analysis based on smoking status, the results showed that the GSTM1 polymorphism was significantly related to NPC risk among smokers $(\mathrm{OR}=3.16,95 \% \mathrm{CI}$ : 1.76-5.67, $P=000)$, but not among nonsmokers ( $\mathrm{OR}=1.01$, 95\% CI: $0.56-1.82, P=0.982$ ) (Table 2 ). In addition, we also performed stratified analysis based on the source of control: it revealed the significant results in population-based studies $(\mathrm{OR}=1.40,95 \%$ CI: 1.19-1.64, $P=000)$ (Table 2).

\section{Sensitivity analysis and bias diagnosis}

In order to compare the difference and evaluate the sensitivity of the meta-analyses, we used both models (the fixed-effects

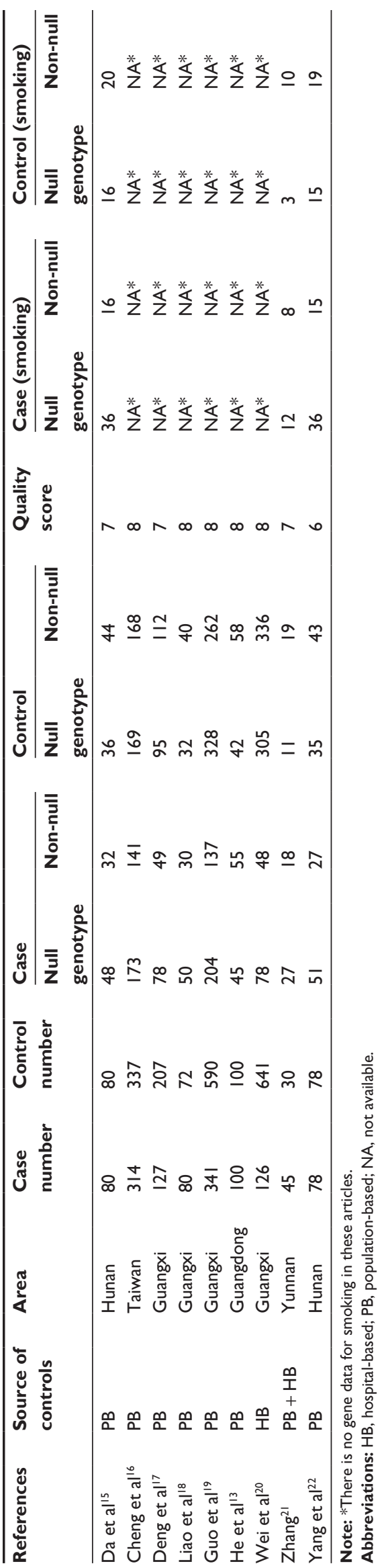




\begin{tabular}{|c|c|c|}
\hline Study ID & OR (95\% Cl) & Weight \% \\
\hline Da et $\mathrm{al}^{15}$ & $1.83(0.98-3.43)$ & 4.75 \\
\hline Cheng et $\mathrm{al}^{16}$ & $1.22(0.90-1.66)$ & 24.14 \\
\hline Deng et $a^{17}$ & $1.88(1.20-2.94)$ & 9.19 \\
\hline Liao et $a^{18}$ & $2.08(1.09-3.99)$ & 4.16 \\
\hline Guo et $\mathrm{al}^{19}$ & $1.19(0.91-1.56)$ & 31.83 \\
\hline He et $\mathrm{al}^{13}$ & $1.13(0.65-1.98)$ & 7.62 \\
\hline Wei et $\mathrm{al}^{20}$ & $1.79(1.21-2.65)$ & 12.59 \\
\hline Zhang $^{21}$ & $2.59(1.00-6.72)$ & 1.74 \\
\hline Yang et $a^{22}$ & $2.32(1.22-4.43)$ & 3.99 \\
\hline Overall $\left(P^{2}=30.9 \%, P=0.171\right)$ & $1.47(1.27-1.70)$ & 100.00 \\
\hline 0.149 & & \\
\hline
\end{tabular}

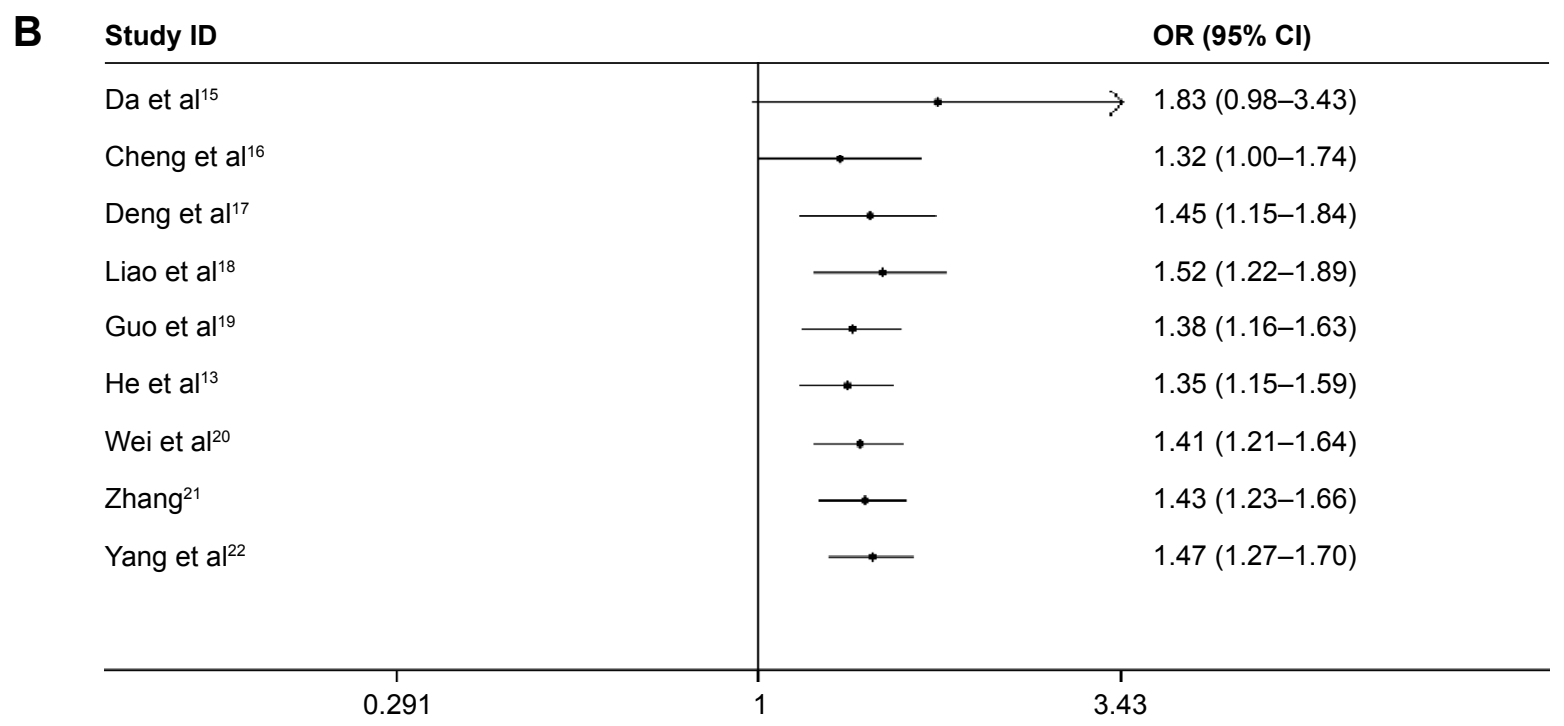

Figure 2 The forest plots of all selected studies on the association between GSTMI polymorphism and NPC risk in South China.

Notes: (A) Meta-analysis; (B) cumulative meta-analysis. Weights are from fixed-effects analysis.

Abbreviations: $\mathrm{Cl}$, confidence interval; NPC, nasopharyngeal carcinoma; OR, odds ratio.

model and the random-effects model) to evaluate the stability of the meta-analysis. All results were not materially altered, except the subgroup analysis for nonsmokers (Table 2). Hence, results of the sensitivity analysis suggest that the data in this meta-analysis are relatively stable and credible. The Begg's funnel plot and Egger's test were performed to assess the publication bias of literatures. The shape of the funnel plots did reveal obvious asymmetry (Figure 3A).

Table 2 Main results in the total and subgroup analysis

\begin{tabular}{|c|c|c|c|c|c|}
\hline \multirow[t]{2}{*}{ Subgroups } & \multirow{2}{*}{$\begin{array}{l}\text { Study } \\
\text { (n) }\end{array}$} & \multirow{2}{*}{$\begin{array}{l}\text { Random-effects model } \\
\text { OR }(95 \% \mathrm{Cl})\end{array}$} & \multirow{2}{*}{$\begin{array}{l}\text { Fixed-effects model } \\
\text { OR }(95 \% \mathrm{CI})\end{array}$} & \multicolumn{2}{|c|}{ Heterogeneity } \\
\hline & & & & $\overline{\chi^{2}}$ & $P$-value \\
\hline Total analysis & 9 & $1.54(1.28-1.86)$ & $1.47(1.27-1.70)$ & 11.58 & 0.171 \\
\hline \multicolumn{6}{|l|}{ Source of control } \\
\hline Population-based & 7 & $1.47(1.19-1.80)$ & $1.40(1.19-1.64)$ & 8.86 & 0.182 \\
\hline \multicolumn{6}{|l|}{ Smoking } \\
\hline Smokers & 3 & $3.15(1.75-5.66)$ & $3.16(1.76-5.67)$ & 0.40 & 0.818 \\
\hline Nonsmokers & 3 & $1.01(0.55-1.83)$ & $1.01(0.56-1.82)$ & 0.88 & 0.645 \\
\hline
\end{tabular}

Note: Data shown in bold represent significant results.

Abbreviations: $\mathrm{Cl}$, confidence interval; $\mathrm{OR}$, odds ratio. 
A

Funnel plot with pseudo $95 \%$
confidence limits

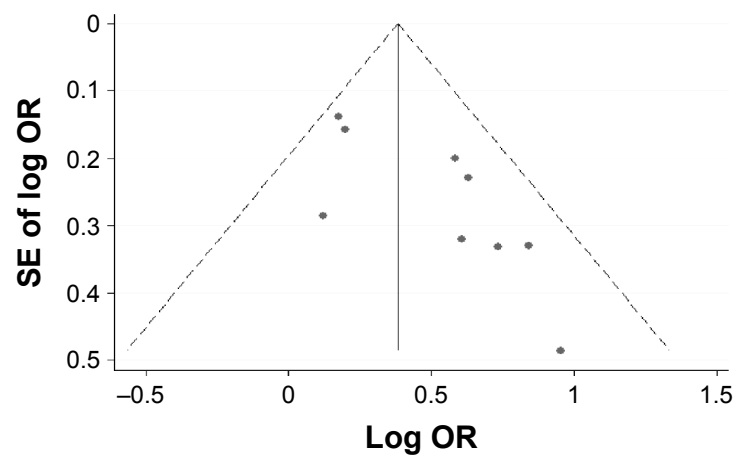

B

Egger's publication bias plot

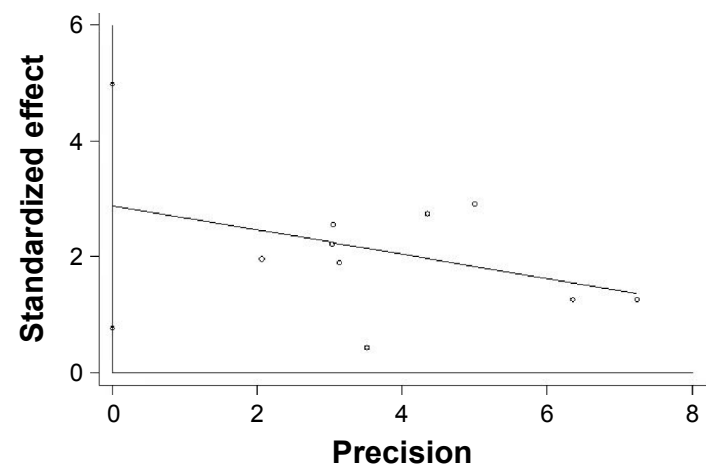

Figure 3 Publication bias assessment of GSTMI polymorphism and NPC risk in Chinese. Notes: (A) Begg's funnel plot, (B) Egger's test.

Abbreviations: SE, standard error; NPC, nasopharyngeal carcinoma; OR, odds ratio.

Then, the Begg's and Egger's tests were used to provide statistical evidence of funnel-plot symmetry. The Begg's and Egger's tests indicated that there was obvious publication bias in overall analyses (Figure 3B, $Z=2.29, P=0.022 ; t=3.23$, $P=0.014)$.

\section{Discussion}

This meta-analysis aimed to systematically summarize the epidemiological evidence for the association between GSTM1 null genotype and risk of NPC in Chinese. Nine case-control studies with 1,291 NPC cases and 2,135 controls were finally included in the meta-analysis. The metaanalysis of a total of nine studies showed that GSTM1 null genotype was significantly associated with an increased risk of NPC in South China (Figure 2A). The findings from the cumulative meta-analysis showed that there was a trend of more obvious association between GSTM1 null genotype and risk of NPC in Chinese from South China based on data accumulated by publication year (Figure 2B). Therefore, GSTM1 null genotype is significantly associated with an increased risk of NPC in South China. The findings from this meta-analysis provide new and strong epidemiological evidence for the association between GSTM1 null genotype and risk of NPC in Chinese. To date, four meta-analyses have been published, assessing the association between GSTM1 polymorphism and NPC risk, but the existing evidence was still weak due to limited sample size in Chinese populations, ethnic difference, or disagreements among the published studies. ${ }^{8-11}$ To our knowledge, our study represented the first meta-analysis with a large sample size, studying the interaction of GSTM1 variant with NPC in the Chinese population. Therefore, the present meta-analysis of all available casecontrol studies was conducted to shed some light on those inconsistent results.
Furthermore, the interaction between GSTM1 null genotype and tobacco smoking for the risk of NPC has been evaluated by several studies with inconsistent results, possibly because of small sample size. ${ }^{8}$ Thus, we performed another analysis stratified by smoking to ascertain the interaction by pooling all available studies. We found that the GSTM1 null genotype significantly increased the risk of NPC in smokers. In addition, results of the sensitivity analysis suggest that the data are stable and credible. We concluded that the association between GSTM1 null genotype and NPC risk was strongest in individuals with exposure to smoking. Moreover, the association between the extent of smoke exposure and NPC risk was not clear; further studies with larger sample size are needed to provide insights into the interaction association. When we performed the subgroup analyses comparing with control groups, significant association with susceptibility for the development of NPC was found in population-based studies. There might be some explanations for the significant association. First, gene-gene or gene-environmental interactions might play an important role in susceptibility to NPC. In addition, decades of epidemiological studies have shown that NPC has unique prevalence features, including regional, racial, and familial aggregation. ${ }^{3}$

The pathways of carcinogen metabolism are complex, mediated by the activities of multiple genes. The effect of any single gene might have a limited impact on NPC risk than have so far been anticipated. NPC has some known major environmental determinants other than Epstein-Barr virus infection, and large studies with detailed exposure information are needed to evaluate reliably any moderate genetic effects. Otherwise, some limitations should be acknowledged. First, all studies included in this meta-analysis were in South China, because of the limited data in North China. Some inevitable biases may exist. Second, our results were 
based on unadjusted estimates. More precise analyses can be conducted if individual data were available, which would allow for the adjustment by other covariates including age, sex, race, and other factors. Finally, because the papers searched in our study were limited to those openly published, it is possible that some related unpublished studies that may meet the inclusion criteria were missed. Hence, a publication bias was evident in Begg's and Egger's tests in relation to GSTM1 null genotype, which may interfere with the interpretation of the results. However, a careful search of published studies and subgroup analyses were conducted to minimize this.

\section{Conclusion}

In conclusion, our meta-analysis supports that GSTM1 null genotype might contribute to individual susceptibility to NPC in South China. In the future, more studies on gene-gene and gene-environment interactions are required. Such studies taking these factors into account may eventually lead to a better understanding of the effect of GSTM1 null genotype in the development of NPC.

\section{Disclosure}

The authors report no conflicts of interest in this work.

\section{References}

1. Jemal A, Bray F, Center MM, Ferlay J, Ward E, Forman D. Global cancer statistics. CA Cancer J Clin. 2011;61:69-90.

2. Yu WM, Hussain SS. Incidence of nasopharyngeal carcinoma in Chinese immigrants, compared with Chinese in China and South East Asia: review. J Laryngol Otol. 2009;123:1067-1074.

3. Cao SM, Simons MJ, Qian CN. The prevalence and prevention of nasopharyngeal carcinoma in China. Chin J Cancer. 2011;30:114-119.

4. O'Neil JD, Owen TJ, Wood VH, et al. Epstein-Barr virus-encoded EBNA1 modulates the AP-1 transcription factor pathway in nasopharyngeal carcinoma cells and enhances angiogenesis in vitro. J Gen Virol. 2008; 89:2833-2842.

5. Soya SS, Vinod T, Reddy KS, Gopalakrishnan S, Adithan C. Genetic polymorphisms of glutathione-S-transferase genes (GSTM1, GSTT1 and GSTP1) and upper aerodigestive tract cancer risk among smokers, tobacco chewers and alcoholics in an Indian population. Eur J Cancer. 2007; 43:2698-2706.

6. Hayes JD, Flanagan JU, Jowsey IR. Glutathione transferases. Annu Rev Pharmacol Toxicol. 2005;45:51-88.

7. McIlwain CC, Townsend DM, Tew KD. Glutathione S-transferase polymorphisms: cancer incidence and therapy. Oncogene. 2006;25: $1639-1648$

OncoTargets and Therapy

\section{Publish your work in this journal}

OncoTargets and Therapy is an international, peer-reviewed, open access journal focusing on the pathological basis of all cancers, potential targets for therapy and treatment protocols employed to improve the management of cancer patients. The journal also focuses on the impact of management programs and new therapeutic agents and protocols on
8. Sun ZF, Zhang J, Xu HM, Wang GL, Dong P. Association between GSTM1 polymorphism and nasopharyngeal cancer susceptibility: a meta-analysis. Asian Pac J Cancer Prev. 2012;13(11):5817-5821.

9. Murthy AK, Kumar V, Suresh KP. Meta-analysis of GSTM1 and GSTT1 polymorphisms and risk of nasopharyngeal cancer. Asian Pac J Cancer Prev. 2013;14(3):1697-1701.

10. Wei Y, Zhou T, Lin H, et al. Significant associations between GSTM1/ GSTT1 polymorphisms and nasopharyngeal cancer risk. Tumour Biol. 2013;34(2):887-894.

11. Zhuo X, Cai L, Xiang Z, Li Q, Zhang X. GSTM1 and GSTT1 polymorphisms and nasopharyngeal cancer risk: an evidence-based metaanalysis. J Exp Clin Cancer Res. 2009;28:46.

12. Wells GA, Shea B, O'Connell D, et al. The Newcastle-Ottawa Scale (NOS) for Assessing the Quality of Nonrandomized Studies in Meta-Analyses. Available from: www.ohri.ca/programs/clinical_epidemiology/oxford. htm. Accessed July 28, 2015.

13. He Y, Zhou GQ, Li X, Dong XJ, Chai XQ, Yao KT. 谷胱甘肽硫转 移酶M1基因编码区多态性与中国南方人群鼻咽癌易感性的矢系 [Correlation of polymorphism of the coding region of glutathione S-transferase M1 to susceptibility of nasopharyngeal carcinoma in South China population]. Ai Zheng. 2009;28(1):5-7. Chinese.

14. Jiang Y, Li N, Dong P, et al. Polymorphisms in GSTM1, GSTTI and GSTP1 and nasopharyngeal cancer in the east of China: a case-control study. Asian Pac J Cancer Prev. 2011;12(11):3097-3100.

15. Da SJ, Liang B, Wu HL, et al. GSTM1基因多态性与鼻咽癌遗传易感 性的矢系研究 [Relationship between GSTM1 gene polymorphism and genetic susceptibility in nasopharyngeal carcinoma]. Prac J Cancer. 2002;17:617-626. Chinese.

16. Cheng YJ, Chien YC, Hildesheim A, et al. No association between genetic polymorphisms of CYP1A1, GSTM1, GSTT1, GSTP1, NAT2, and nasopharyngeal carcinoma in Taiwan. Cancer Epidemiol Biomarkers Prev. 2003;12:179-180.

17. Deng ZL, Wei YP, Luo W, et al. Glutathione S-transferase M1 and T1 gene deletion associated with increased susceptibility to nasopharyngeal carcinoma. Chin German J Clin Oncol. 2005;4:276-278.

18. Liao ZL, Deng ZL, Wei YP, et al. GSTT1, GSTM1 基因缺失多态 性与鼻咽癌的发病矢系 [Relationship of GSTT1 and GSTM1 gene polymorphisms with the development of nasopharyngeal carcinoma]. J Guangxi Med Univ. 2005;22:372-374. Chinese.

19. Guo X, O'Brien SJ, Zeng Y, Nelson GW, Winkler CA. GSTM1 and GSTT1 gene deletions and the risk for nasopharyngeal carcinoma in Han Chinese. Cancer Epidemiol Biomarkers Prev. 2008;17:1760-1763.

20. Wei YP, Long XD, Liu ZG, et al. Genetic polymorphism of glutathioneS-transferase M1 and T1 in associated with carcinogenesis of hepatocellular carcinoma and nasopharyngeal carcinoma. Chin German J Clini Oncol. 2012;11:138-141.

21. Zhang G. CYP1A1及 BSTM1 基因多态性与鼻咽癌发病风险矢系的 研究 [To Investigate the Relationship between CYP1A1 and GSTM1 Gene Polymorphisms and Nasopharyngeal Carcinoma [thesis]]. Dali, People's Republic of China: Dali University; 2012. Chinese.

22. Yang J, Zhu SH, Yang ZF, Mo Q. GSTM1 基因多态性与鼻咽癌 遗传易感性研究 [Association between GSTM1 polymorphism and nasopharyngeal cancer susceptibility]. Chin Health Care Nutr. 2014;3:1781-1782. Chinese.

patient perspectives such as quality of life, adherence and satisfaction The manuscript management system is completely online and includes a very quick and fair peer-review system, which is all easy to use. Visit http://www.dovepress.com/testimonials.php to read real quotes from published authors. 